\title{
Politique
}

\section{Les répondants-discrets et l'élection québécoise de 1985}

\section{Guy Lachapelle}

Numéro 10, automne 1986

Opinions et votes

URI : https://id.erudit.org/iderudit/040533ar

DOI : https://doi.org/10.7202/040533ar

Aller au sommaire du numéro

Éditeur(s)

Société québécoise de science politique

ISSN

0711-608X (imprimé)

1918-6584 (numérique)

Découvrir la revue

Citer cet article

Lachapelle, G. (1986). Les répondants-discrets et l'élection québécoise de 1985. Politique, (10), 31-54. https://doi.org/10.7202/040533ar d'utilisation que vous pouvez consulter en ligne.

https://apropos.erudit.org/fr/usagers/politique-dutilisation/ 


\title{
LES RÉPONDANTS-DISCRETS ET L'ÉLECTION QUÉBÉCOISE DE 1985
}

\author{
Guy Lachapelle* \\ Université Concordia
}

«L'attention aux refus de répondre qui sont souvent considérés comme de petits détails d'enquête, donne en réalité beaucoup plus d'informations sur les modes de production des opinions et, partant, sur les conditions d'une utilisation rigoureuse des sondages, que bien des commentaires sur leur signification politique».

L'élection québécoise du 2 décembre 1985 aura retenu l'attention des sondeurs principalement à cause de deux phénomènes; soit une sous-estimation des intentions de vote en faveur du Parti libéral et une sur-estimation de ces dernières à l'endroit des tierspartis. Plusieurs hypothèses ont été suggérées par les politicologues pour expliquer ces écarts (Blais, Crête, Lachapelle, 1986; Lachapelle, 1985) entre les intentions de vote et le vote réel le soir du 2 décembre. Parmi celles-ci, l'on peut mentionner le calcul de la marge d'erreur, le taux de réponse, la formulation des questions concernant les intentions de vote, les difficultés d'échantillonnage et les méthodes de répartition des indécis ${ }^{1}$. L'objectif de la présente

* L'auteur tient à remercier André Blais ainsi que trois évaluateurs anonymes pour leurs commentaires.

1. Le terme d'indécis a plusieurs significations dans la littérature en science politique. Ici nous entendons tous les répondants à un sondage qui n'indiquent aucune préférence à l'endroit de l'un des partis politiques en lice. Il s'agit donc de ceux qui refusent de répondre, ne savent pas, annulent ou ne votent pas. Ces répondants sont 
étude n'est pas d'évaluer la pertinence de chacune de ces explications car il est souvent difficile d'obtenir des maisons de sondage tous ces renseignements mais plutôt de présenter un modèle permettant de mieux préciser les caractéristiques socio-économiques des répondants-discrets ${ }^{2}$ tout en identifiant quelle méthode de répartition des répondants-discrets aurait été la meilleure dans le contexte de l'élection de décembre 1985. Ceci nous permettra d'expliquer pourquoi les intentions de vote en faveur du Parti libéral ont été sous-estimées et celles en faveur des tiers-partis sur-estimées.

La répartition proportionnelle des indécis dans les sondages représente la méthode la plus couramment employée, sinon la seule, par les maisons de sondage au Québec, surtout dans le cas des sondages électoraux. Cette méthode est relativement simple et efficace puisqu'il s'agit de répartir les indécis suivant les caractéristiques des répondants-indiscrets, c'est-à-dire de ceux qui ont affiché une préférence à l'endroit de l'un des partis politiques en liste. Ainsi, retrouve-t-on généralement dans les média les résultats des intentions de vote sans répartition et après répartition proportionnelle des indécis. Le tableau 1 présente ces résultats pour les treize sondages électoraux rendus publics au cours de la dernière campagne électorale. À partir de ces données, l'on peut noter que suivant les résultats moyen des sondages le Parti libéral aurait dû normalement obtenir, après répartition proportionnelle des indécis, un pourcentage de voix aux environs de $49.9 \%$, le Parti québécois $41.3 \%$ et les tiers-partis $8.5 \%$. Si l'on compare

habituellement regroupés dans la catégorie «autres réponses» à la question sur les intentions de vote. Toutefois, l'on peut difficilement définir ceux qui indiquent qu'ils annuleront ou ne voteront pas comme des «indécis" puisqu'ils affichent clairement une opinion.

2. Nous définissons les répondants-discrets comme étant les répondants à un sondage qui refusent de répondre ou ne savent pas. Nous préférons également le terme répondants-discrets au concept de discrets puisqu'il permet de bien préciser que l'étude porte sur les répondants et non pas sur les non-répondants dont on sait en général peu de choses. Quant aux répondants-indiscrets, ils se différencient des répondants-discrets du fait qu'ils manifestent leur appui à l'un des partis politiques. 


\section{TABLEAU 1}

Résultats des sondages de la campagne électorale québécoise de 1985 sans répartition et après répartition proportionnelle des indécis ${ }^{1}$.

\begin{tabular}{|c|c|c|c|c|c|c|c|c|}
\hline \multirow{2}{*}{$\begin{array}{l}\text { Maisons } \\
\text { de } \\
\text { sondage }\end{array}$} & \multirow[b]{2}{*}{ Période } & \multicolumn{4}{|c|}{$\begin{array}{c}\text { Sans répartition } \\
\%\end{array}$} & \multicolumn{3}{|c|}{$\begin{array}{c}\text { Répartition } \\
\text { proportionnelle } \\
\%\end{array}$} \\
\hline & & PLQ & PQ & Tiers-partis & Indécis (2) & PLQ & $\mathrm{PQ}$ & Tiers-partis \\
\hline SORECOM & $11-20$ oct. & 39 & 32 & 6 & 23 & 50 & 41 & 9 \\
\hline CROP & $22-28$ oct. & 40 & 37 & 6 & 16 & 48 & 45 & 7 \\
\hline IQOP & $24-28$ oct. & 37 & 31 & 9 & 23 & 48 & 40 & 12 \\
\hline SORECOM & 28-31 oct. & 37 & 30 & 10 & 23 & 48 & 39 & 13 \\
\hline CREATEC + & 4-7 nov. & 43 & 36 & 5 & 16 & 51 & 43 & 6 \\
\hline SORECOM & 4-11 nov. & 39 & 32 & 7 & 23 & 50 & 42 & 8 \\
\hline CROP & 8-10 nov. & 42 & 37 & 6 & 15 & 50 & 44 & 6 \\
\hline JOLICOEUR & 6-18 nov. & 37 & 33 & 8 & 22 & 48 & 42 & 10 \\
\hline SORECOM & 11-19 nov. & 42 & 35 & 6 & 16 & 51 & 42 & 7 \\
\hline IQOP & 18-22 nov. & 38 & 31 & 8 & 23 & 50 & 40 & 10 \\
\hline CROP & $22-24$ nov. & 44 & 32 & 6 & 18 & 54 & 39 & 7 \\
\hline SORECOM & 21-26 nov. & 43 & 35 & 8 & 14 & 50 & 41 & 9 \\
\hline CREATEC + & $25-27$ nov. & 42 & 32 & 8 & 18 & 51 & 39 & 10 \\
\hline \multicolumn{2}{|c|}{$\begin{array}{l}\text { Moyennes } \\
\text { Résultats de } \\
\text { l'élection }\end{array}$} & 40.2 & 33.3 & 7.1 & 19.2 & $\begin{array}{l}49.9 \\
56.0\end{array}$ & $\begin{array}{l}41.3 \\
38.7\end{array}$ & 8.8 \\
\hline
\end{tabular}

(1) Les données proviennent des journaux qui ont publié les résultats.

(2) Les indécis représentent ici les répondants qui ne savent pas ou refusent de répondre (les répondants-discrets) et ceux qui ne voteront pas ou annuleront. 
ces chiffres avec le vote réel obtenu par les partis politiques suite à l'élection, on observe des écarts importants: le vote pour le Parti libéral est sous-estimé de $7.1 \%$ tandis que le vote pour le Parti québécois et les tiers-partis est sur-estimé de $2.7 \%$ et $3.1 \%$ respectivement.

Cependant, dans la mesure où l'on reconnaît qu'un sondage est avant tout le reflet de l'opinion publique à un moment précis, il serait sans aucun doute plus juste d'étudier uniquement les résultats des trois derniers sondages soit ceux qui ont été réalisés après le débat radiophonique francophone des chefs. C'est ce que nous avons fait dans un autre article (Blais, Crête, Lachapelle, 1986) en regardant de plus près les résultats de ces trois sondages après répartition des indécis suivant les méthodes suggérées par les diverses maisons de sondage. Ainsi, si l'on compare ces trois sondages, c'est-à-dire ceux de CROP, SORECOM et CREATEC+, on peut immédiatement observer que les écarts sont sensiblement réduits. Après le débat francophone des chefs, $51.7 \%$ des répondants affirment qu'ils voteront pour le Parti libéral le jour de l'élection, $39.6 \%$ favorisent le Parti québécois et $8.7 \%$ les tierspartis. Conséquemment, les écarts précédemment observés sont considérablement réduits, la sous-estimation des intentions de vote en faveur du Parti libéral n'étant plus que de $4.3 \%$ et la sur-estimation des intentions de vote à l'endroit du Parti québécois n'étant que de $0.9 \%$. Toutefois, les intentions de vote en faveur des tiers-partis sont en moyenne plus élevées ce qui accroît davantage la sur-estimation de leurs intentions de vote, l'écart étant maintenant de $3.4 \%$. Malgré tout, la sous-estimation des intentions de vote en faveur du Parti libéral et la sur-estimation de ces dernières à l'endroit des tiers-partis demeurent et sont supérieures à la marge d'erreur agrégée qui est pour ces trois derniers sondages de $2.0 \%$ (2528 répondants). Les écarts entre les prévisions des sondeurs et les résultats du vote, sans être énormes, vont donc au-delà des marges d'erreurs habituelles. 
Mais la question qui se pose ici est de savoir si la méthode utilisée pour répartir les indécis, soit la répartition proportionnelle, constitue une méthode adéquate pour prédire le vote. La première limite de cette méthode, telle qu'employée par les maisons de sondage, est qu'aucune distinction n'est faite parmi les indécis entre les répondants qui affirment qu'ils ne savent pas pour qui voter ou refusent d'indiquer leurs intentions de vote (les répondantsdiscrets) et ceux qui disent qu'ils annuleront ou ne voteront pas.

Une seconde méthode de répartition des indécis fut également proposée lors de la dernière campagne électorale par la firme SORECOM, soit une répartition «7-2» des indécis en faveur du Parti libéral. Ainsi, si l'on répartit les résultats du dernier sondage SORECOM (du 21 au 26 novembre) suivant cette méthode on obtient $53.0 \%$ des intentions de vote en faveur du Parti libéral, $39.0 \%$ au Parti québécois et $8.0 \%$ pour les tiers-partis. Malheureusement, cette procédure de répartition n'est ni justifiée, ni expliquée et cela est d'autant plus surprenant que cette maison de sondages n'y avait pas eu recours dans les sondages antérieurs.

Une troisième méthode a également été déjà proposée par André Bernard à partir d'une étude systématique des divers sondages électoraux publiés au Québec depuis le début des années soixantedix. Ainsi, Bernard remarque que de façon générale les sondages politiques ont de manière générale sous-estimé de $" 5$ points de pourcentage en moyenne, le vote réel du Parti libéral» (Bernard et Descôteaux, 1981, 96).

Ces trois méthodes ne sont certes pas les seules qui ont été jusqu'à présent proposées. Récemment, nous proposions une répartition des indécis suivant la formule 60\% au Parti libéral, $32 \%$ au Parti québécois et $8 \%$ aux tiers-partis (Blais, Crête, Lachapelle, 1986, tableau 4). Mais la difficulté majeure avec toutes ces méthodes est que leur fondement théorique reste souvent ambigu et repose davantage sur l'intuition et l'analyse de tendances observables que sur de solide base empirique. L'objectif de la 
présente étude est justement de combler ces lacunes tout en répondant à certaines interrogations concernant les répondants-discrets, c'est-à-dire ceux et uniquement les répondants qui indiquent qu'ils ne savent pas pour qui voter ou refusent de répondre à la question concernant les intentions de vote. Nous chercherons ainsi à identifier les variables qui ont été les plus déterminantes dans le choix des électeurs québécois lors du scrutin de décembre 1985 et qui permettent de prévoir le vote réel. Puis, à partir de ces résultats, nous établirons qu'elle méthode de classification des répondants-discrets semble la plus adéquate. Comme nous l'avons déjà mentionné, nous nous limiterons à l'étude des répondantsdiscrets car nous croyons que ceux-ci ont un comportement différent des électeurs qui répondent lors des sondages qu'ils s'abstiendront ou annuleront. En somme, notre but premier n'est pas de formuler une théorie de l'opinion publique ${ }^{3}$ mais simplement d'élaborer une méthode qui permet de mieux répartir les répondants-discrets entre les divers partis politiques.

\section{Les caractéristiques des répondants-discrets}

Certaines hypothèses ont été proposées au sujet des répondantsdiscrets et leurs caractéristiques socio-économiques bien que les données demeurent très parcellaires. Comme nous l'avons vu deux postulats ont été établis concernant les répondants-discrets qui ont une incidence directe sur le choix du type de méthode de répartition de ces derniers. L'on estime généralement soit que les répondants-discrets devraient avoir un comportement électoral assez semblable à celui des répondants-indiscrets, d'où leur redistribution suivant la méthode proportionnelle, soit qu'ils aient des caractéristiques sociologiques qui les rapprochent des électeurs

3. Sur la définition du concept d'opinion et l'ambiguité entourant la notion de "sondages d'opinion" voir l'article de Pierre Bourdieu, "L'opinion publique n'existe pas", Les Temps Modernes, 29: 318 (janvier 1973): 1292-1309. 
libéraux d'où la nécessité de sur-estimer leur distribution en faveur du Parti libéral suivant les méthodes "plus cinq et moins cinq", «-2» ou «60-32-8».

Deux hypothèses sont au moins suggérées pour justifier ce rapprochement répondants-discrets - Parti libéral. Les répondantsdiscrets sont assez semblables aux électeurs libéraux du fait qu'ils sont, tout comme ces derniers, plus âgés et moins scolarisés. Les résultats du sondage Jolicœur - Le Devoir - Radio-Québec ${ }^{4}$ confirment ces hypothèses. Ainsi observe-t-on un plus grand pourcentage de répondants-discrets dans le groupe des 65 ans et plus que dans tout autre groupe d'âge; plus on vieillit plus la propension à être répondant-discret s'accentue. À l'inverse, plus le niveau de scolarité est faible plus le pourcentage de répondants-discrets augmente; l'on retrouve ainsi $29.5 \%$ de répondants-discrets parmi les répondants ayant entre 0 et 7 ans de scolarité, $21.2 \%$ chez ceux ayant entre 8 et 11 ans de scolarité, $18.0 \%$ chez ceux avec plus de 16 années de scolarité. Dans ces deux cas, l'on observe les mêmes tendances chez les répondants libéraux à savoir qu'ils sont règle générale plus âgés et moins scolarisés (voir tableaux 2 et 3).

De plus, dans plusieurs sondages, l'on a souvent observé le fait que le nombre de femmes discrètes étaient supérieur au nombre d'hommes. Ce phénomène ne semble pas propre à la situation québécoise puisque Bourdieu parle d'un phénomène assez général (Bourdieu, 1973: 1926 et 1977: 60) non seulement propre à la société française mais également observable dans d'autres contextes socio-politiques. Dans le sondage Jolicœur - Le Devoir - RadioQuébec, l'on retrouve parmi les répondants-discrets quelques

4. Dansla présente étude, nous utiliserons ce sondage afin d'analyser le comportement des répondants-discrets. Ce sondage a été réalisé entre le 6 et le 18 novembre 1985; l'échantillon-maître comprenait 1598 cas et le taux de réponse fut de $63.5 \%$. Nous tenons principalement ici à remercier André Blais et Jean Crête pour nous avoir permis d'utiliser les données de ce sondage. 


\section{TABLEAU 2}

Résultats croisés entre les intentions de vote et l'âge.

\begin{tabular}{lccccc}
\hline & \multicolumn{5}{c}{ Âge } \\
\cline { 2 - 6 } \multicolumn{1}{c}{$\begin{array}{c}\text { Les intentions } \\
\text { de vote }\end{array}$} & $18-25$ & $26-40$ & $41-64$ & 65 et + & Total \\
\hline Parti libéral & 130 & 201 & 202 & 53 & 588 \\
& $(39.0)$ & $(41.2)$ & $(45.2)$ & $(48.2)$ & $(42.5)$ \\
Parti québécois & 157 & 209 & 133 & 21 & 520 \\
& $(47.2)$ & $(42.4)$ & $(29.7)$ & $(19.1)$ & $(37.6)$ \\
Répondants-discrets & 46 & 81 & 112 & 36 & 275 \\
& $(13.8)$ & $(16.4)$ & $(25.1)$ & $(32.7)$ & $(19.0)$ \\
Total & 333 & 483 & 447 & 110 & 1383 \\
& $(100.0)$ & $(100.0)$ & $(100.0)$ & $(100.0)$ & $(100.0)$ \\
\hline
\end{tabular}

TABLEAU 3

Résultats croisés entre les intepontions de vote et la scolarité.

\begin{tabular}{lccccc}
\hline \multirow{2}{*}{$\begin{array}{c}\text { Les intentions } \\
\text { de vote }\end{array}$} & $0-7$ & $8-11$ & $12-15$ & 16 et + & Total \\
\cline { 2 - 6 } & 72 & 193 & 245 & 73 & 583 \\
Parti libéral & $(45.3)$ & $(45.8)$ & $(40.6)$ & $(38.0)$ & $(42.4)$ \\
& 40 & 139 & 250 & 90 & 519 \\
Parti québécois & $(25.2)$ & $(33.0)$ & $(41.4)$ & $(46.9)$ & $(37.7)$ \\
& 47 & 89 & 109 & 29 & 274 \\
Répondants-discrets & $(29.5)$ & $(21.2)$ & $(18.0)$ & $(15.1)$ & $(19.9)$ \\
& 159 & 421 & 604 & 192 & 1376 \\
Total & $(100.0)$ & $(100.0)$ & $(100.0)$ & $(100.0)$ & $(100.0)$ \\
& & & & & \\
\hline
\end{tabular}

$61.0 \%$ de femmes contre seulement $39.0 \%$ d'hommes. L'on remarque également chez les répondants-discrets une confiance plus faible dans les chefs des deux principales formations politiques, le Parti libéral et le Parti québécois, que les répondants-indiscrets bien que $37.7 \%$ ont davantage confiance en Johnson, $17.0 \%$ en Bourassa et $13.3 \%$ ont une confiance partagée à l'égard de 
ces deux leaders. Conséquemment, même si les répondants-discrets sont peut-être plus près des répondants libéraux, ils semblent malgré tout avoir une plus grande confiance dans le chef du Parti québécois.

Mais toutes ces observations demeurent très descriptives car elles ne nous permettent pas d'identifier précisément les caractéristiques particulières des répondants-discrets. Il ne faut pas oublier que les caractéristiques des répondants-discrets peuvent varier d'une élection à l'autre. Tout ce que ces éléments apportent c'est de confirmer certaines tendances observées précédemment et ce sans donner des explications à ces divers phénomènes. De plus, tout ceci n'explique pas véritablement pourquoi certains écarts demeurent entre les sondages et les résultats de la dernière élection car, même dans l'hypothèse où tous les répondants-discrets nonfrancophones ont voté pour le Parti libéral, ce dernier ne récolterait que $10.0 \%$ de plus d'appuis chez les répondants-indiscrets, ce qui n'améliorait son vote, sur l'ensemble des intentions de vote, que de 1 pour cent (Blais, Crête, Lachapelle, 1986). Dès lors, si l'identification des caractéristiques des répondants-discrets permet à tout le moins d'observer quelques tendances, elle ne permet toutefois pas de préciser quelles variables demeurent les plus importantes pour expliquer les intentions de vote ou le comportement de ces répondants-discrets. Il nous faut d'autres méthodes pour mieux saisir toute la portée de ces phénomènes.

\section{Remarques préliminaires et méthodologie}

Afin d'identifier les variables clés qui permettent de mieux prévoir les intentions de vote des répondants-discrets, nous avons procédé à une analyse discriminante (Klecka, 1980, 1975, 1973) dans le but d'établir dans un premier temps les caractéristiques socio-économico-politiques des répondants-indiscrets et dans un second temps comment celles-ci contribuent à expliquer les intentions 
de vote de ces derniers. Grâce à ces résultats, nous serons en mesure de prédire le comportement des répondants-discrets.

Pour les fins de cette analyse, nous avons utilisé le sondage Jolicœur - Le Devoir - Radio-Québec réalisé entre le 6 et le 18 novembre 1985 . Ce sondage a plusieurs qualités dont celle d'avoir été, de tous les sondages publiés durant la campagne électorale celui qui avait le nombre le plus élevé de répondants, soit $1598^{5}$; tous les autres sondages ont eu en moyenne environ 1000 répondants. Ceci nous permet donc d'avoir un plus grand nombre de répondants-discrets, soit 278 ou $17.4 \%$ de l'échantillon; de ce nombre, $179(11.2 \%)$ indiquaient ne pas savoir pour quel parti voter et $99(6.2 \%)$ refusaient d'indiquer leur intention de vote.

Ce sondage utilisait deux questions afin de connaître les intentions de vote des répondants. D'abord, on leur demandait:

Q18. Si une élection provinciale avait lieu aujourd'hui, pour lequel des partis suivants voteriez-vous?

Il s'agissait ici d'une question fermée où les noms de quatre partis politiques étaient mentionnés dans l'ordre suivant: le Parti québécois, le Parti libéral, le Parti conservateur, le Nouveau Parti démocratique ou pour un autre parti. Dans ce dernier cas, le répondant devait identifier le parti pour lequel il avait l'intention de voter. Suite à cette question, le nombre de répondants-discrets était de 392, soit 304 ne savent pas et 88 refus. Puis, l'on posait la question suivante aux 304 personnes ayant indiqué qu'ils ne savaient pas pour quel parti voter:

5. De l'échantillon initial de 1598 cas, 80 répondants n'ont pas été retenu lors de l'analyse. De ce nombre, 46 indiquèrent leur intention de s'abstenir, 27 annuleraient et 7 n'avaient pas droit de vote. Notre échantillon final était donc de 1518 répondants. 
Q19. Votre décision n'est peut-être pas encore prise. Malgré cela, quel est le parti que vous êtes tenté d'appuyer présentement?

Suite à cette seconde question dont l'objectif est d'inciter les répondants-discrets à indiquer une certaine préférence, seul 190 répondants demeurèrent des répondants-discrets: 179 ne savaient toujours pas pour quel parti voter et 11 refusèrent d'indiquer une préférence. Parmi les autres répondants-discrets à la première question, 110 devinrent des répondants-indiscrets, indiquant une préférence pour l'un des partis, et 4 affirmèrent qu'ils n'avaient pas droit de vote ${ }^{6}$.

Dès lors, la somme des questions 18 et 19 constitue le total des intentions de vote pour les divers partis. Ainsi 590 répondants $(36.9 \%)$ indiquèrent leur préférence pour le Parti libéral, 520 (32.6\%) pour le Parti québécois, 130 (8.2\%) pour un tiersparti et $278(17.4 \%)$ demeurèrent des répondants-discrets. 80 autres répondants indiquèrent leur intention de s'abstenir, d'annuler ou n'avaient pas droit de vote $(4.9 \%)$. L'objet de cette étude étant d'étudier les répondants-discrets, nous nous intéresserons donc à ces 278 répondants qui n'indiquèrent aucune intention de vote.

L'analyse discriminante que nous vous présentons ici se fera en deux étapes. Premièrement, nous élaborerons un modèle où

6. Il semble que le type de questions posées par les maisons de sondage pour connaître les intentions de vote constitue un facteur important suivant qu'elles optent pour une approche ouverte ou fermée. Ainsi, Guy Trudel, du Centre de sondage de l'Université de Montréal, observait dans le cas des élections québécoises que "lorsque l'interviewer énumère les partis politiques, la proportion des indécis est moins importante que si les partis ne sont pas mentionnés: on passe de $23.1 \%$ à $12.6 \%$ d'indécis, une différence significative» (Trudel, 1984). Si cette explication peut nous permettre de mieux comprendre certains écarts observés dans les sondages québécois du début des années soixante-dix cela n'explique cependant pas les résultats des sondages de la dernière campagne électorale où toutes les maisons de sondages ont utilisé, à notre connaissance à tout le moins, l'approche fermée avec quelques variantes suivant que l'on posait une ou deux questions afin de connaître l'intention de vote des sondés. 
certaines variables clées permettant d'expliquer les intentions de vote des répondants-indiscrets seront identifiées. Puis, deuxièmement, il s'agira d'analyser chacun des répondants-discrets et de prévoir leur vote en comparant leurs caractéristiques avec celles des répondants-indiscrets. Ce type d'analyse a donc un double objectif: l'un d'interprétation du comportement électoral des répondants-indiscrets et l'un de classification des répondants-discrets à partir des résultats obtenus au sujet des premiers (Klec$\mathrm{ka}, 1980$, 9). Ce type d'analyse postule donc que les répondantsdiscrets ayant des caractéristiques semblables à celles des répondantsindiscrets voteront de la même façon que ceux-ci, tout en séparant les répondants-discrets qui demeurent de véritables discrets. Ainsi, l'analyse discriminante nous permettra-t-elle, par l'utilisation d'une série de prédictions (les variables discriminantes), de distinguer au maximum ce qui différencie les répondants indiquant une intention de vote à l'endroit du Parti libéral, du Parti québécois, ou d'un tiers-parti, et de classifier par la suite les répondantsdiscrets à l'intérieur de l'un de ces trois groupes.

\section{Résultats et analyse}

La présentation des résultats de notre analyse discriminante se fera en deux étapes. D'abord, nous identifierons les variables qui permettent le mieux de distinguer parmi les répondantsdiscrets les intentions de vote à l'endroit du Parti libéral, du Parti québécois ou d'un tiers-parti. Initialement, plusieurs variables socio-économiques ont été sélectionnées, telles que le revenu, l'âge, la profession, le sexe, le niveau de scolarité pour ne nommer que ceux-là. Toutefois, l'avantage de l'analyse discriminante est de ne retenir que les variables qui permettent le mieux de différencier deux ou plusieurs types d'électeurs. Une fois cette première étape franchie, soit d'identifier le modèle dont le pouvoir discriminant est le plus élevé, nous serons en mesure de classifier les répondants- 
discrets dans chacune des trois catégories de vote retenues (Parti libéral, Parti québécois, tiers-partis). Nous procéderons donc à une analyse discriminante tri-dimensionnelle dans une première étape ${ }^{7}$.

\section{L'analyse tri-dimensionnelle}

Quatre variables discriminantes ont été incluses dans notre modèle qui permettent de distinguer de manière relativement satisfaisante les répondants-indiscrets favorables au Parti libéral, au Parti québécois et aux-tiers-partis. Il s'agit du degré de satisfaction à l'endroit du gouvernement du Parti québécois, du degré de confiance dans les leaders du Parti québécois et du Parti libéral, de la langue maternelle et du niveau de scolarité. Le tableau 4 présente l'ensemble des variables retenues dans le modèle ainsi que l'importance relative de chacune des fonctions. L'utilisation des résultats standardisés nous permet d'évaluer la contribution de chacune de nos variables à l'intérieure de chaque fonction (Klecka, 1980: 29) tandis que les coefficients structuraux (ceux entre parenthèses dans le tableau 4) nous permettent de mieux saisir le pouvoir discriminant de chacune des variables entre les trois groupes retenus. Dans le cas des coefficients standardisés, leur interprétation est relativement facile puisqu'ils ont la même signification que des coefficients de régression (Myers et O'Connor, 1983, 424-425) bien qu'il faut ignorer leur direction (Klec$\mathrm{ka}, 1980,30$ ). Ainsi, plus la valeur d'un coefficient standardisé

7. Toutes les variables que nous avons utilisés sont des variables dichotomiques. Bien que l'analyse discriminante est particulièrement désignée pour le traitement de variables intervalles continues, l'utilisation de quelques variables dichotomiques ou de plusieurs peut être justifiée de la même façon que dans une analyse de régression. C'est du moins la conclusion à laquelle nous sommes arrivés à la lumière des travaux de Klecka (1973) des analyses de Fenwick et al. (1982) et de Myers (1983.

Deux postulats sont par ailleurs importants dans toute analyse discriminante à savoir l'absence de collinéarité entre variables et l'uniformité des matrices de covariance entre groupes. Nos résultats ne semblent pas déroger à ces règles. 
est élevée plus la contribution de la variable sera importante à l'intérieur d'une fonction.

Selon les résultats présentés au tableau 4 , deux fonctions peuvent être identifiées; une première appellée péquistes et une seconde à laquelle nous avons donné le nom de libéraux. En ce qui concerne les péquistes, l'on peut noter que le degré de confiance dans le chef du Parti québécois représente la variable la plus importante de cette fonction suivi dans l'ordre par le degré de confiance envers le chef du Parti libéral, le degré de satisfaction à l'endroit du gouvernement du Parti québécois, le fait d'être de langue maternelle française, un degré de scolarité élevé et d'être de langue maternelle anglaise. Dans le cas de ces deux dernières variables, on peut affirmer qu'elles ont un rôle relativement secondaire à l'intérieur de la fonction péquiste puisque la valeur de leur coefficient est en dessous de 0.20 (Myers et O'Connor, 1983, 425).

Pour ce qui est de la deuxième fonction, soit les libéraux, on remarque également le rôle déterminant du degré de confiance envers le chef du Parti libéral, M. Robert Bourassa, suivi par le degré de confiance envers Pierre-Marc Johnson, le degré de satisfaction envers le gouvernement du Parti québécois, le fait d'être de langue maternelle anglaise, d'avoir un haut niveau de scolarité et finalement d'être de langue maternelle française. De toutes ces variables, seule la dernière, soit d'être de langue maternelle française joue un rôle secondaire. Conséquemment, les coefficients standardisés nous révèlent le rôle majeur joué par le degré de confiance envers les chefs et le niveau de satisfaction à l'endroit du gouvernement du Parti québécois dans les intentions de vote des péquistes et des libéraux.

Toutefois, pour saisir l'effet véritable de chacune de ces variables à l'intérieur d'une fonction et leur pouvoir discriminant, il faut regarder de plus près les coefficients structuraux. Ainsi, observet-on chez les péquistes un degré de confiance élevé à l'endroit de 
TABLEAU 4

Analyse discriminante tri-dimensionnelle des répondants-indiscrets ${ }^{1}$.

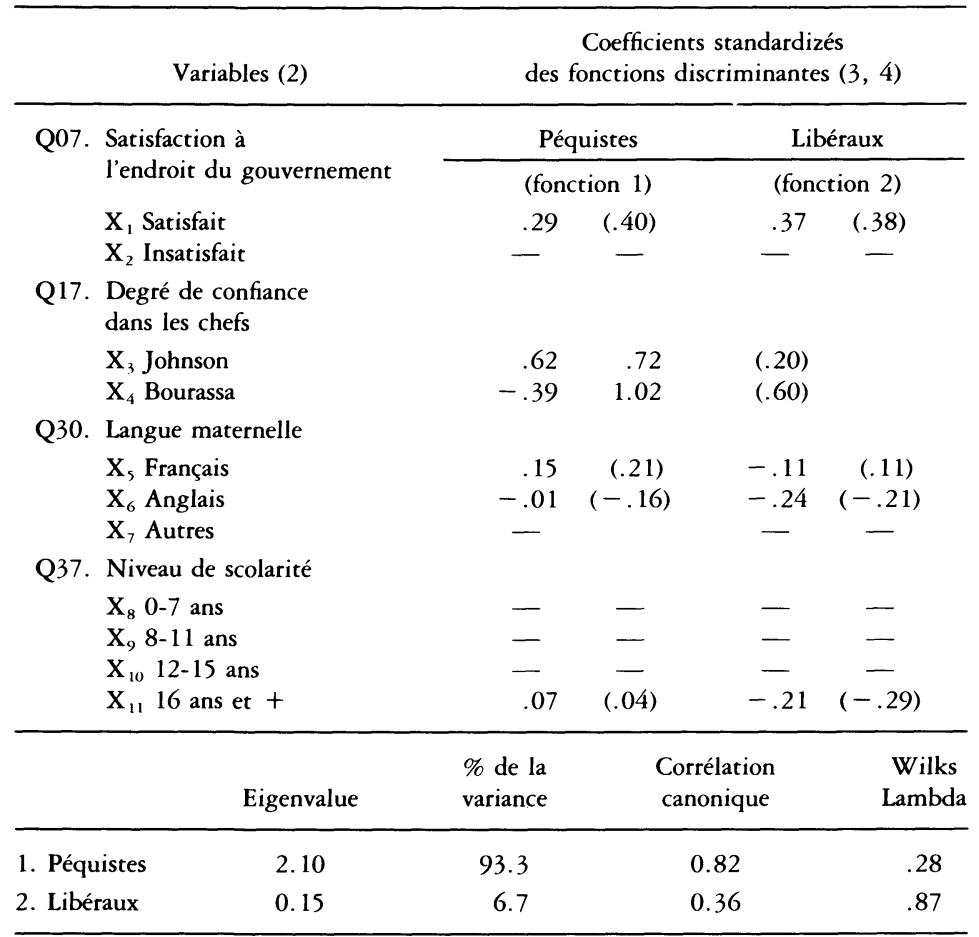

(1) Pour les fins de la présente étude nous avons utilisé le programme SPSS d'analyse discriminante.

(2) Il s'agit des variables qui ont été retenues lors de l'analyse; d'autres variables indépendantes ont été utilisées lors de l'analyse prélininaire, telles que l'âge et le sexe, mais leur contribution au modèle était négligeable.

(3) Comme il s'agit d'une analyse tri-dimensionnelle puisque nous cherchons à différencier trois groupes, soit ceux qui indiquent leur intention de voter pour le Parti libéral, le Parti québécois ou un tiers-parti $(\mathrm{N}=3)$, le nombre de fonctions discriminantes est égal à $\mathbf{N}-1$. Deux fonctions ont donc été identifiées. le niveau de tolérance fut fixé à .005 et la méthode discriminante utilisée fut celle du V de Rao.

$\mathrm{La}$ valeur des coefficients pour les variables dont le $\mathrm{F}$ était inférieur à 1.0 ne sont pas rapportés.

(4) Le premier coefficient est celui qui est standardizé tandis que celui entre parenthèses nous indique l'importance relative des variables de chaque fonction à l'intérieur de chaque groupe ("within-groups structure coefficients»). 
Pierre-Marc Johnson $(0,87)$ et un manque évident de confiance envers le chef du Parti libéral ( -0.77$)$. De plus, l'on note chez eux un degré de satisfaction également assez élevé envers le gouvernement du Parti québécois. Finalement, le fait d'être de langue maternelle française joue aussi un rôle important dans les intentions de vote des péquistes. Quant au fait d'être de langue maternelle anglaise, bien qu'on puisse penser que cette variable permet d'exclure un répondant comme étant un péquiste, il faut être prudent car le pouvoir discriminatoire de cette variable est très faible tout comme celui d'avoir un niveau de scolarité élevé.

Par contre, chez les libéraux, l'on observe aussi un degré de confiance relativement élevé envers le chef du Parti libéral, Robert Bourassa, mais moindre que celui des péquistes envers le chef du Parti québécois. Par ailleurs, l'on remarque assez paradoxalement un degré de satisfaction élevé chez les libéraux envers le gouvernement du Parti québécois. De plus, le fait d'avoir un niveau de scolarité élevé et d'être de langue maternelle anglaise sont deux éléments qui ne semblent pas (signes négatifs) caractéristiques des libéraux. Finalement, l'on remarque chez ceux-ci un faible degré de confiance envers le chef du Parti québécois et le fait d'être de langue maternelle française n'a qu'un rôle minime. En somme, ce que traduit cette seconde fonction c'est sans doute toute l'ambiguité de nombre d'électeurs lors de l'élection de décembre 1985 qui tout en préférant le chef du Parti libéral continuaient d'avoir un degré de satisfaction assez élevé envers le gouvernement du Parti québécois mais une confiance mitigée envers le nouveau chef de cette formation politique.

Lorsque l'on observe la valeur discriminante de ces deux fonctions pour identifier les intentions de vote (voir tableau 4), l'on note le pouvoir supérieur de la première fonction. Ainsi, l'eigenvalue de cette première fonction est-il quatorze fois supérieur à celui de la seconde fonction. En d'autres termes, la fonction péquiste contient à elle seule $93.3 \%$ du pouvoir discriminant 
total de notre modèle. Le lambda de Wilks traduit également l'utilité de cette fonction pour différencier les intentions de vote des répondants-indiscrets. Toutefois, la seconde fonction, bien que son rôle soit moindre, n'a toutefois pas un rôle négligeable puisque le coefficient de corrélation canonique indique une association moyenne entre les groupes et cette fonction discriminante.

\section{Le degré de prévision de notre modèle}

Le second objectif de cette analyse, après avoir déterminé quelles variables pouvaient le mieux différencier les intentions de vote des répondants-indiscrets, est de préciser la valeur prévisionnelle de notre modèle. Ainsi, au tableau 5, l'on peut noter que les

\section{TABLEAU 5}

Classification des répondants-indiscrets.

\begin{tabular}{lcccc}
\hline & \multicolumn{4}{c}{ Valeur prévisionnelle du modèle (1) } \\
\cline { 2 - 5 } Intention de vote & $\begin{array}{c}\text { Parti } \\
\text { libéral }\end{array}$ & $\begin{array}{c}\text { Parti } \\
\text { québécois } \\
\mathrm{N}(\%)\end{array}$ & $\begin{array}{c}\text { Tiers-parti } \\
\mathrm{N}(\%)\end{array}$ & $\begin{array}{c}\text { Total } \\
\mathrm{N}\end{array}$ \\
\hline Parti libéral & 399 & 50 & 69 & 517 \\
Parti québécois & $(17.1)$ & $(9.6)$ & $(13.3)$ & 476 \\
Tiers-parti & 12 & 443 & 21 & 105 \\
& $(2.5)$ & $(93.1)$ & $(4.4)$ & 48 \\
& 11 & 46 & $(45.7)$ & $1098(2)$ \\
\hline
\end{tabular}

(1) Ce tableau se lit comme suit: 399 des 517 répondants qui ont indiqué leur intention de voter pour le Parti libéral ont été correctement classifiés par notre modèle, 50 se rapprochaient davantage des caractéristiques des voteurs péquistes, et 69 de celles des voteurs des tiers-partis. Le degré de prédiction de notre modèle est de $81.1 \%$ $(399+443+48 / 1098)$.

(2) 142 répondants-indiscrets accusent une valeur manquante pour au moins l'une des variables discriminantes. 
deux fonctions de notre modèle peuvent prévoir les intentions de vote des répondants-indiscrets dans $81.1 \%$ des cas.

Notre modèle peut toutefois prévoir avec plus d'acuité les intentions de vote en faveur du Parti québécois puisque $93.2 \%$ des répondants-indiscrets qui ont indiqué leur préférence pour ce parti sont correctement classés. Chez les répondants libéraux, la valeur prévisionnelle de notre modèle est moindre, se situant à $77.1 \%$, tandis qu'elle est relativement faible dans le cas des tiers-partis, de $45.7 \%$ seulement. Mais ce qui est tout aussi intéressant dans ces résultats, c'est d'observer que des 517 répondantsindiscrets qui ont indiqué leur préférence à l'endroit du Parti libéral, 69 avaient des caractéristiques davantage similaires à celles des répondants favorables à un tiers-parti et 50 se rapprochaient davantage des répondants péquistes. Par ailleurs, chez les répondants-indiscrets qui ont indiqué leur préférence pour un tiersparti, soit un total de 105 répondants, 46 ressemblent bien plus aux péquistes et 11 aux libéraux. Ce que ces résultats traduisent c'est sans doute le fait que bon nombre de répondants-indiscrets, bien que favorables au Parti québécois ou parce qu'ils l'avaient appuyé lors d'élections précédentes, hésitaient au moment de la campagne électorale de 1985 à réaffirmer leur soutien à ce parti et préféraient indiquer leur intention de voter pour un tiers-parti. L'on peut penser dans ce dernier cas aux employés du secteur public. Toutefois, ce que ces chiffres semblent montrer c'est que ceux qui avaient l'intention de voter pour le Nouveau parti démocratique et le Parti conservateur (des 130 répondants du sondage favorables à un tiers-parti, 58 indiquèrent leur intention de voter N.P.D. et 42 P.C.) ont des caractéristiques sociologiques qui s'apparentent bien plus aux répondants-indiscrets qui ont affiché leur préférence pour le Parti québécois et le Parti libéral. Bien que la première explication n'exclut pas nécessairement la seconde, il n'en demeure pas moins que notre modèle a certes plus de 
difficulté à classifier adéquatement les répondants libéraux et ceux des tiers-partis que les répondants péquistes.

\section{Classifucation des répondants-discrets}

Notre dernière tâche, et sans doute la plus importante, consiste à évaluer comment notre modèle distribuent les répondantsdiscrets à l'intérieur des trois groupes retenus pour l'analyse, soit le Parti libéral, le Parti québécois et les tiers-partis. L'objectif premier est ici de prévoir, compte tenu des caractéristiques des répondants-indiscrets et de l'apport de diverses variables pour expliquer les intentions de vote de ces derniers, comment les répondants-discrets devraient se comporter, en d'autres termes de déterminer lequel des partis politiques les répondants-discrets sont le plus susceptibles d'appuyer.

Comme on peut l'observer au tableau 6 , notre modèle a classé $52.3 \%$ des répondants-discrets du sondage Jolicœur — Le Devoir - Radio-Québec avec les répondants-indiscrets favorables à un tiers-parti, 30.1\% avec ceux qui indiquèrent leur intention de voter pour le Parti québécois et $17.6 \%$ avec le groupe favorable au Parti libéral. Ces résultats indiquent donc clairement que les répondants-discrets ressemblent davantage aux répondants-indiscrets qui ont indiqué une préférence à l'endroit d'un tiers-parti. Il est par ailleurs assez surprenant d'observer qu'un nombre plus élevé de répondants-discrets s'apparentent aux électeurs favorables au Parti québécois en comparaison avec ceux ayant des caractéristiques sociologiques relativement semblables à celle des partisans libéraux. Notre analyse discriminante nous avait déjà révélé cette tendance entre libéraux et péquistes et si l'on postule que les répondantsdiscrets se rapprochant des répondants favorables à un tiers-parti voteront pour le Parti libéral, l'on se retrouverait avec $69.9 \%$ des répondants-discrets favorables au Parti libéral et $30.1 \%$ qui voteraient pour le Parti québécois. 


\section{TABLEAU 6}

Classification des répondants-discrets.

\begin{tabular}{lccc}
\hline & $\begin{array}{c}\text { Intention de vote des } \\
\text { répondants- } \\
\text { indiscrets } \\
\text { Parti }(\%)\end{array}$ & $\begin{array}{c}\text { Répondants } \\
\text { discrets classifiés par le } \\
\text { modèle }\end{array}$ & $\begin{array}{c}\text { Total } \\
\text { N }(\%)\end{array}$ \\
\hline Parti libéral & 517 & N $\%)$ & 551 \\
Parti québécois & $(47.1)$ & 34 & $(42.7)$ \\
Tiers-parti & 476 & $(17.6)$ & 534 \\
& $(43.3)$ & 58 & $(41.4)$ \\
Total & 105 & $(30.1)$ & 206 \\
& $(9.6)$ & 101 & $(15.9)$ \\
& 1098 & $(52.3)$ & 1291 \\
& $(100.0)$ & 193 & $(100.0)$ \\
\hline
\end{tabular}

(1) 85 répondants-discrets accusent une valeur manquante pour au moins l'une des variables discriminantes.

(2) Au total, 227 répondants ont été exclus de l'analyse à cause de valeurs manquantes $(1518-227=1291)$.

Aussi, pour évaluer la pertinence d'une telle prévision dans l'hypothèse où une certaine polarisation du vote en faveur des deux principales formations politiques se produit à la fin d'une campagne électorale, nous avons procédé à une analyse discriminante bi-dimensionnelle. Comme nous l'avons mentionné précédemment, les sondages ont en général tendance à sur-estimer les intentions de vote en faveur des tiers-partis et à sous-évaluer la performance du Parti libéral. Ainsi, en redistribuant le vote des répondantsdiscrets vers les deux principales formations politiques en présence, l'on pourra déterminer quelle méthode de répartition serait à première vue la plus adéquate, et ce bien sûr dans le contexte de l'élection québécoise de 1985.

Le tableau 7 présente les résultats de cette analyse discriminante bi-dimensionnelle où les mêmes variables que celles de notre 
premier modèle ont été utilisées ${ }^{8}$. D'abord, l'on peut noter que le fait de restreindre notre second modèle à deux seuls groupes et conséquemment à une seule fonction permet d'augmenter le degré de prévision à $91.3 \%$. Ainsi, $93.7 \%$ des répondantsindiscrets favorables au Parti québécois sont adéquatement classés et $89.0 \%$ de ceux favorisants le Parti libéral. Par ailleurs, $62.2 \%$ des répondants-discrets auraient probablement appuyés le Parti libéral contre $37.8 \%$ pour le Parti québécois. Ce que nos résultats suggèrent c'est qu'une répartition des répondants-discrets suivant la méthode «62-38» aurait certainement été la meilleure formule dans le contexte précis de l'élection de décembre 1985. Conséquemment, l'on peut noter que la méthode suggérée ici s'apparente à celles proposées par d'autres politicologues bien qu'elle repose

\section{TABLEAU 7}

Résultats de l'analyse bi-dimensionnelle.

\begin{tabular}{lccc}
\hline & \multicolumn{3}{c}{ Valeur prévisionnelle du modèle (1) } \\
\cline { 2 - 4 } Intention de vote & $\begin{array}{c}\text { Parti libéral } \\
\mathrm{N}(\%)\end{array}$ & $\begin{array}{c}\text { Parti québécois } \\
\mathrm{N}(\%)\end{array}$ & $\begin{array}{c}\text { Total } \\
\mathrm{N}(\%)\end{array}$ \\
\hline Parti libéral & 460 & 57 & 517 \\
& $(89.0)$ & $(11.0)$ & 476 \\
Parti québécois & 30 & 446 & 143 \\
Répondants-discrets & $(6.3)$ & $(93.7)$ & \\
& 89 & $(37.8)$ & 1136 \\
\hline
\end{tabular}

(1) Le degré de prédiction de ce modèle est de $91.3 \%$

8. Comme dans notre premier modèle, le degré de confiance à l'endroit de Pierre-Marc Johnson, le degré de satisfaction élevé envers le gouvernement du Parti québécois et le faible degré de confiance vis-à-vis de Robert Bourassa sont les variables les plus importantes. En d'autres termes, ces trois variables permettent de différencier les répondants péquistes des répondants libéraux et de prédire comment ils voteront le jour de l'élection. 
sur une étude plus systématique des répondants-indiscrets tout en représentant un appui empirique à ce que d'autres avaient pu concevoir de manière intuitive.

\section{Conclusions}

Cette étude ne s'est intéressée qu'aux répondants-discrets, soit ceux qui ont refusé d'indiquer leur intention de vote ou qui ne savaient pas pour quel parti voter au moment où le sondage Jolicœur - Le Devoir - Radio Québec fut réalisé. La même méthodologie pourrait également être employée dans le cas des répondants qui ont indiqué leur intention de s'abstenir ou d'annuler leur vote. Toutefois, dans la mesure où ces derniers ne constituent pas au sens strict des «indécis», ils devraient avoir un comportement différent des répondants-discrets. Aussi, toutes les méthodes de répartition des «indécis» ont-elles cette limite soit de considérer toute "autre réponses" comme étant le résultat de l'indécision.

Ce que notre modèle démontre c'est qu'une formule de redistribution des répondants-discrets sur la base «62-38» aurait été sans aucun doute plus adéquate dans le contexte de l'élection québécoise de 1985 et ce par rapport aux autres méthodes proposées. Ainsi, l'utilisation de cette formule, au lieu de la méthode proportionnelle, pour répartir les quelques $17.4 \%$ de répondantsdiscrets du sondage Jolicœur — Le Devoir - Radio-Québec améliore la performance du Parti libéral de $1.4 \%$. Suivant la méthode proportionnelle, le Parti libéral aurait recueilli $47.6 \%$ des voix contre $49.0 \%$ avec la formule «62-38». Conséquemment, le Parti québécois aurait reçu $40.5 \%$ des voix au lieu de $42.0 \%$ et les tiers-partis $10.4 \%$. On demeure malgré tout loin des résultats de l'élection et la formule proposée ne résout en rien le problème de la sur-estimation du vote aux tiers-partis.

Bien que l'analyse discriminante présente des avantages certains pour différencier et classifier des groupes de répondants, il serait 
sans doute présomptueux de croire que l'on peut trouver une méthode qui satisfasse à toutes les élections où d'entretenir, comme certains sondeurs le font (Francœur, 1986, 92), le mythe que seule la compréhension du comportement électoral des indécis permet de gagner ou de perdre une élection. Les sondages demeurent et demeureront un événement politico-sociologique et notre étude a démontré que même dans le cas des répondants-indiscrets on ne peut pas être tout à fait certain de leur intention de vote. Enfin, il faut être prudent puisque les résultats obtenus ici l'on été avec un seul sondage réalisé à un moment précis de la campagne électorale. Pour obtenir des généralisations jouissant d'une plus grande validité, il faudra vérifier la pertinence de ce modèle avec d'autres sondages.

\section{RÉFÉRENCES}

BERNARD, André et DESCÔTEAUX, Bernard, Québec : élections 1981, (Montréal : Hurtubise H.M.H., 1981).

BLAIS, André, CRÊTE, J. et LACHAPELLE, G., «L'élection québécoise de 1985: un bilan des sondages", Revue canadienne de science politique, 19: 2 juin 1986): 325336.

BOURDIEU, Pierre, "Questions de politique», Actes de la recherche en sciences sociales, 16 (septembre 1977), 55-91.

BOURDIEU, Pierre, «L'opinion publique n'existe pas», Les Temps Modernes, 29: 318 (janvier 1973), 1292-1309.

COLOMB, Philippe, «Les non-réponses aux questions d'opinion sur la politique de population", Population, 32: 4-5 (juillet-octobre 1977), 835-866.

DE SINGLY, François, «La gestion sociale des silences», Consommation, 29: 4 (octobredécembre 1982), 37-63.

FENWICK, Ian, WISEMAN, F., BECKER, J. F. et HEIMAN, J. R., "Classifying Undecided Voters in Pre-election Polls», The Public Opinion Quarterly, 46: 3 (automne 1982), 383-391.

FERBER, Robert, "Item Nonresponse in a Consumer Survey", The Public Opinion Quarterly, 30: 3 (automne 1966), 399-415.

FRANCIS, Joe D. et BUSCH, Lawrence, "What We Now Know About «I Don't Knows", The Public Opinion Quarterly, 39: 2 (été 1975), 207-218.

FRANCOEUR, Louis-Gilles, "Comment Robert Bourassa a «organisé» Pierre-Marc Johnson", L'Actualité, 11: 2 (février 1986), 88, 90, 92.

GAXIE, Daniel, Le sens caché - Inégalités culturelles et ségrégation politique, (Paris: Editions du Seuil, 1978). 
GERGEN, Kenneth J. et BACK, KURT W., "Communication in the Interview and the Disengaged Respondent », The Public Opinion Quarterly, 30 : 3 (automne 1966), 385-398.

GLENN, Norval D., "Aging, Disengagement, and Opinionation», The Public Opinion Quarterly, 33: 1 (printemps 1969), 17-23.

KLECKA, William R., Discriminant Analysis, (Sage University Papers, Series on Quantitative Applications in the Social Sciences, 07-019, Beverly Hills, CA, Sage Publications, 1980).

KLECKA, William R., "Discriminant Analysis", dans Norman N. Nie et al., SPSS, (New York: McGraw-Hill Inc. 1975), 434-467.

KLECKA, William R., "The clientele of Australian parties: New perspectives through discriminant analysis", Politics, 7: 2 (novembre 1973), 301-308.

LACHAPELLE, Guy, «Les indécis et la «spirale du silence», Le Devoir 76: 285 (9 décembre 1985), 1, 8.

MICHELAT, Guy et SIMON, Michel, «Les «sans-réponses» aux questions politiques», Pouvoirs, 33 (1985), 41-56.

MICHELAT, Guy et SIMON, Michel, "Systèmes d'opinions, choix politiques, caractéristiques socio-démographiques: résultats d'une analyse typologique", Revue française de science politique, 24: 1 (février 1974), 5-32.

MYERS, David J. et O'CONNOR, Robert E., "The Undecided Respondent in Mandatory Voting Settings: A Venezuelan Exploration", Western Political Quarterly, 36: 3 (septembre 1983), 420-433.

PORTO VASQUEZ, Fernando, "Les jeux de la précision et du silence», Consommation, 29: 4 (octobre-décembre 1982), 13-35.

TRUDEL, Guy, "Les intentions de vote de l'électorat québécois», Le Devoir, 76: 43 (21 février 1984), 9. 

TABLEAU 4

Analyse discriminante tri-dimensionnelle des répondants-indiscrets ${ }^{1}$.

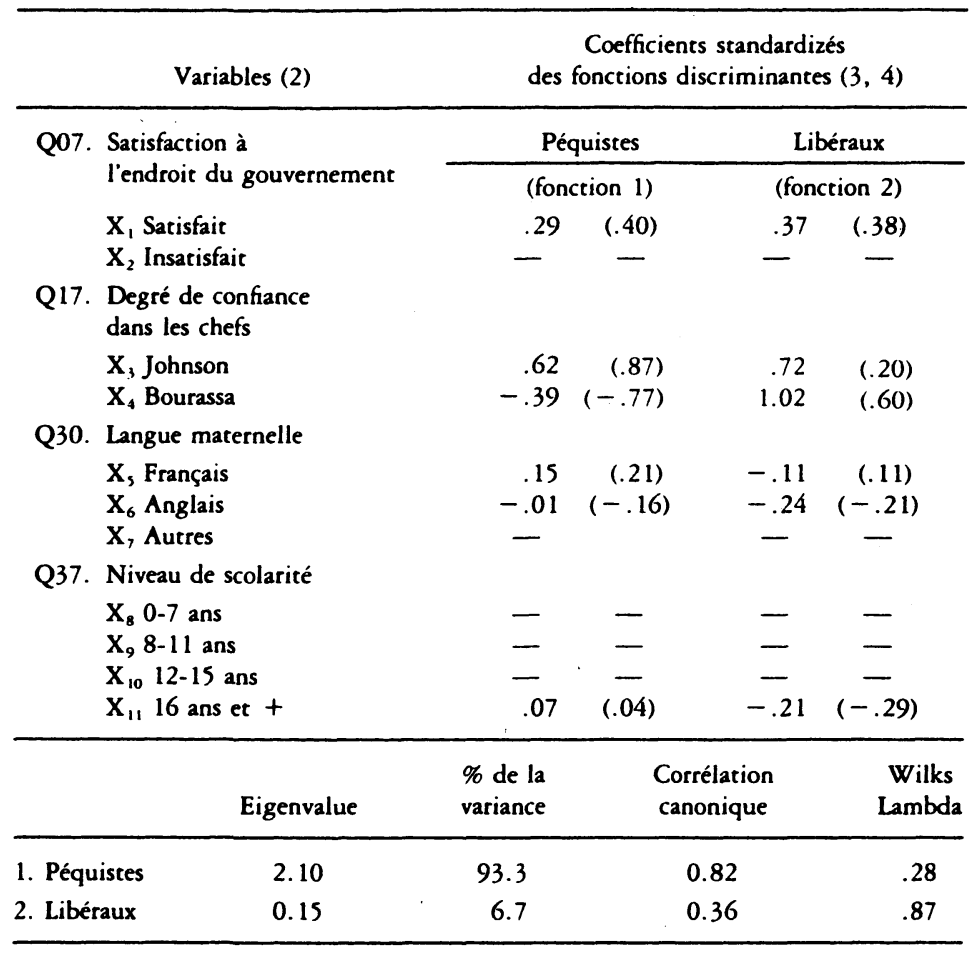

(1) Pour les fins de la présente étude nous avons utilisé le programme SPSS d'analyse discriminante.

(2) Il s'agit des variables qui ont été retenues lors de l'analyse; d'autres variables indépendantes ont été utilisées lors de l'analyse prélininaire, telles que l'âge et le sexe, mais leur contribution au modèle était négligeable.

(3) Comme il s'agit d'une analyse tri-dimensionnelle puisque nous cherchons à différencier trois groupes, soit ceux qui indiquent leur intention de voter pour le Parti libéral, le Parti québécois ou un tiers-parti $(\mathrm{N}=3)$, le nombre de fonctions discriminantes est égal à $\mathbf{N}-1$. Deux fonctions ont donc été identifiées. le niveau de tolérance fut fixé à .005 et la méthode discriminante utilisée fut celle du V de Rao.

La valeur des coefficients pour les variables dont le $F$ était inférieur à 1.0 ne sont pas rapportés.

(4) Le premier coefficient est celui qui est standardizé tandis que celui entre parenthèses nous indique l'importance relative des variables de chaque fonction à l'intérieur de chaque groupe («within-groups structure coefficients"). 